\title{
Severe Sepsis
}

National Cancer Institute

\section{Source}

National Cancer Institute. Severe Sepsis. NCI Thesaurus. Code C111915.

Clinical syndrome defined by the presence of both infection and a systemic inflammatory response that progresses to multi-organ failure. 